
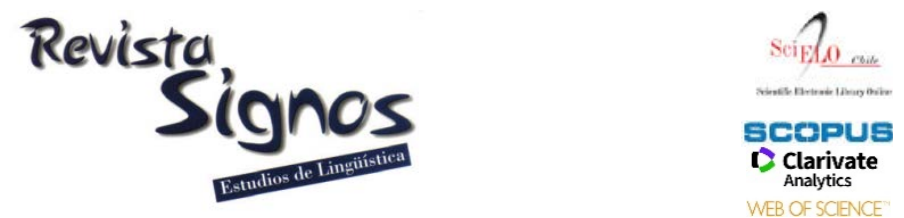

\title{
Procesos experienciales en narraciones sobre situaciones cotidianas escritas por escolares básicos ${ }^{1}$
}

\section{Experiential processes in narratives about daily situations written by primary schools students}

\author{
Manuel Rubio \\ Universidad de SANTIAGo De ChiLE \\ CHILE \\ manuel.rubio@usach.cl
}

Recibido: 05-VII-2016 / Aceptado: 19-I-2018

DOI: $10.4067 /$ S0718-09342019000100158

\section{Resumen}

El propósito de este artículo es describir los procesos representados en las cláusulas finitas que componen las narraciones escritas por escolares de educación básica. Para tal efecto, se analizan el verbo y los grupos verbales como elementos centrales en la configuración de la experiencia, núcleo que se expande con los participantes inherentes al proceso representado y con adjuntos de tipo circunstancial. Se considera la cláusula como unidad de procesamiento central, pues en ella se concretan las elecciones de los hablantes en diferentes redes sistémicas y se las estructura sintagmáticamente. El enfoque metodológico corresponde a una descripción cuantitativa. El corpus está compuesto por 180 textos escritos de escolares de $3^{\circ}, 5^{\circ}$ y $7^{\circ}$ básicos de dos tipos de establecimiento escolares: uno municipal y uno particular subvencionado, con el fin de explorar si existen posibles diferencias. Se observa que en el corpus predominan los procesos materiales que conforman la experiencia como evento externo. No obstante, dicha tendencia va progresivamente disminuyendo a medida que se avanza en la escolaridad, pues aumenta la cantidad de cláusulas con procesos relacionales atributivos y cláusulas mentales. Se concluye que los escolares describen eventos concretos claramente perceptibles desde un punto de vista externo, los cuales tienden a localizar en el tiempo y el espacio.

Palabras Clave: Representación de la experiencia, metafunción experiencial, tipos de proceso, producción escrita, narración escrita. 


\begin{abstract}
The purpose of this article is to describe the processes represented in finite clauses which are part of written narratives produced by elementary school students. In order to achieve this objective, verbs and verb phrases are analyzed as central elements in the configuration of experience, which is expanded by inherent participants in the represented process and circumstantial-type adjuncts. Clauses are considered the central process unit, as they convey the speakers' choice in terms of the different systemic networks that are syntagmatically structured. The methodological approach corresponds to a quantitative description. The corpus presents a collection of 180 texts written by students in Grades 3, 5 and 7 attending one of two types of schools: a state school or a state-subsidized private school. The material processes that construct the experience as an external event, are significantly predominant in the corpus. However, such trend steadily decreases as students are promoted to the next grade, as evidenced by an increase of clauses with attributive and mental relational processes. It can be concluded that students describe concrete events from a perceptively external point of view, which tend to localize time and space.
\end{abstract}

Key Words: Representation of experience, experiential metafunction, types of processes, writing, written narrative.

\title{
INTRODUCCIÓN
}

La lengua es un sistema semiótico social mediante el cual los hablantes pueden establecer distinciones en el flujo de la experiencia, posibilitando su organización, interpretación y comunicación. La comprensión de los fenómenos, la interacción con ellos, su introyección como vivencia personal y compartida con otros, se configura mediante recursos lingüísticos. En otras palabras, el mundo interno y externo requiere ser representado, esto es, configurado mediante la elección de signos verbales codificados como textos (Halliday \& Matthiessen, 1999, 2004).

Desde esta perspectiva, el análisis de la representación de la experiencia requiere entender dicha representación como significado expresado lingüísticamente, es decir, como la concreción de posibilidades que brinda el sistema de la lengua para construir sentido y poder actuar con otros. Indudablemente, el fluir de la experiencia no puede representarse como un todo, sino que requiere ciertas distinciones modeladas como figuras (constituidas por elementos verbales de diferentes clases y que desempeñan diferentes roles) y por secuencia de figuras interrelacionadas entre sí. En términos gramaticales, dichas distinciones se expresan en cláusulas y complejos clausulares que aluden a fenómenos en dominios particulares de la experiencia: los sucesos y acciones en el mundo externo; lo percibido y vivenciado en el mundo de la consciencia; las relaciones de identificación y clasificación de fenómenos; la manifestación externa de procesos subjetivos; las acciones verbales; y el reconocimiento de la existencia de ciertos objetos o eventos (Halliday \& Matthiessen, 1999, 2004). 
La representación de la experiencia varía en grado de complejidad dependiendo de la modalidad de lenguaje utilizado. En efecto, el desarrollo de la habilidad de representar la experiencia por escrito requiere instrucción formal y supone una serie de demandas cognitivas, orientadas a que el niño aprenda a reflexionar sobre la experiencia propia, su conocimiento del mundo, las intenciones deseadas, el despliegue temático y los posibles efectos del texto que produce en otros (Hayes, 1996; Pinheiro \& de Freitas Rossi, 2008; Grabe \& Kaplan, 2014). En otras palabras, la producción escrita implica que el escritor aprenda a gestionar, prototípicamente con ausencia del posible lector, las elecciones lingüísticas que permitan darle forma a su experiencia, construir un texto comprensible, adecuado y efectivo.

No obstante, la importancia de aprender a organizar la experiencia por escrito para comunicarla a otros, son pocos los estudios que exploran los recursos lingüísticos utilizados por los niños a medida que avanzan en la escolaridad (Christie \& Derewianka, 2010). Al parecer, los estudios sobre escritura en educación primaria se han concentrado en el dominio del código escrito (Sotomayor, Molina, Bedwell \& Hernández, 2013; Alegría Ugarte \& Díaz Agüero, 2017), la estructura discursiva (Aravena, 2011; Aravena, Figueroa, Quiroga \& Hugo, 2016; Iparraguirre, Baccala \& Scheuer, 2016), la coherencia (Concha, Aravena, Coloma \& Romero, 2010), la metacognición (Lacon de Lucia \& Ortega de Hocevar, 2008; Crespo, Benítez \& Pérez, 2010), la evaluación de la calidad mediante rúbricas (Benítez, 2009; Benítez \& Sotelo, 2013; Sotomayor, Lucchini, Bedwell, Biedma, Hernández \& Molina, 2013; Sotomayor, Gómez, Jéldrez, Bedwell, Dominguez \& Ávila, 2016) y la complejidad sintáctica (Meneses, Ow \& Benítez, 2012; Aravena \& Hugo, 2016). En otras palabras, prácticamente no existen artículos que tengan como objeto de análisis el uso de recursos verbales empleados por los escolares básicos para representar la experiencia propia o ajena.

En la perspectiva de identificar los recursos léxico-gramaticales para representar la experiencia y su posible enriquecimiento en el curso de la escolaridad básica, este artículo presenta una indagación en los textos escritos por estudiantes de $3^{\circ}, 5^{\circ}$ y $7^{\circ}$ básicos. Para hacerlo, se ha optado por un análisis sobre los procesos representados a nivel clausular, considerando el verbo y los grupos verbales como elementos centrales en la configuración semántica de los fenómenos experimentados. En esta línea, el verbo y los grupos verbales expresan dominios particulares de experiencias y articulan los participantes inherentes al proceso representado. Este centro experiencial (proceso + participantes) puede ser expandido por elementos de tipo circunstancial de carácter temporal, espacial y causal, entre otros (Halliday \& Matthiessen, 2004).

Considerando lo anterior, la finalidad de este artículo es describir los textos escritos por escolares básicos desde el punto de vista de los procesos representados en ellos, analizando las posibles variaciones observadas entre un nivel y otro. Para hacerlo, se configuró un corpus de narraciones escritas en dos establecimientos escolares: uno 
municipal y otro particular subvencionado. Para profundizar la descripción, se exploran las posibles diferencias que hay entre los textos producidos por los estudiantes de estos dos tipos de establecimientos. De este modo, se cuentan con insumos para evidenciar los desempeños posibles de esperar en educación básica.

El artículo parte por una reflexión teórica sobre el rol del lenguaje en la configuración semiótica de la experiencia y su impacto en los procesos de socialización, referencia fundamental para concebir la gramática como un conjunto de opciones paradigmáticas orientadas a la producción de significado. Posteriormente, se detalla el procedimiento para generar el corpus y se ilustra el proceso de segmentación clausular que posibilitó el análisis de los textos. Los resultados se exponen mediante el uso de porcentaje para identificar los procesos predominantes en las cláusulas que componen los textos de los estudiantes, determinar la orientación del relato de cada sujeto, establecer los elementos circunstanciales que expanden los procesos materiales. Asimismo, se describen los cambios que se registran en el uso de cláusulas relacionales y mentales en los estudiantes de los diferentes niveles.

\section{Marco teórico}

\subsection{El lenguaje como semiótica social}

Halliday (1998, 2003) concibe el quehacer lingüístico como construcción e intercambio de significados, proceso que es esencialmente creativo y se encuentra condicionado por el contexto situacional y cultural en que ocurre. Desde este punto de vista, la experiencia es la realidad configurada verbalmente, no la expresión de una estructura conceptual pre-existente codificada lingüísticamente (Halliday \& Matthiessen, 1999). Como recurso simbólico, la lengua no puede reducirse a un sistema de reglas lógicamente estructurado. Por el contrario, su descripción implica develar las opciones que posibilita a sus usuarios para construir y compartir la realidad. En este sentido, las prácticas lingüísticas dentro de una comunidad dada son medios de reflexión y acción sobre los fenómenos vivenciados en diferentes dominios de experiencia. Con dichas prácticas, los individuos configuran sus identidades, posiciones, roles, valores y saberes, simbolizando y tensionando el sistema social compartido, es decir, con el uso de la lengua le dan forma al mundo en que habitan. En consecuencia, la interacción verbal desempeña una función clave en los procesos de socialización familiar y escolar, pues mediante la lengua se transmiten modelos de vida, modos de relación, visiones de mundo y, además, se determina la posición en la estructura social. En definitiva, las prácticas lingüísticas en que se inserta un niño lo configuran como sujeto y lo dotan de recursos para construir y compartir significados (Christie \& Derewianka, 2010; Rose \& Martin, 2012).

La lengua proporciona una serie de patrones sistémicos, de orden paradigmáticos, que expresan las opciones disponibles para la construcción de significados por parte 
del hablante, por ejemplo, la polaridad (positivo o negativo), la especificación de género (niña-niño, criatura), la modulación obligativa, los procesos en el sistema de la transitividad (material, conductual, mental, verbal, relacional, existencial), etc. En este sentido, un texto es el producto de la selección en varios sistemas de redes, elección orientada en función de los propósitos que persiguen los sujetos en su interacción con otros (Eggins, 2003; Halliday \& Matthiessen, 2004; Gutiérrez, 2008). Dichas opciones apuntan a la construcción simultánea de tres tipos de significados sobre: a) el mundo de la experiencia, b) las relaciones interpersonales entre interlocutores y c) la organización textual. Estas opciones se presentan a nivel discursivo, mediante las variables de registro: 'campo' (el tema abordado), 'tenor' (relaciones y roles entre los interlocutores) y 'modo' (la tarea que cumple el lenguaje). A su vez, se reflejan en las cláusulas mediante lo que Halliday denomina 'metafunciones'. La metafunción experiencial se refiere al sistema de la transitividad que representa tanto el mundo físico y de la conciencia como las relaciones abstractas entre eventos como una articulación de procesos, participantes y circunstancias; la metafunción interpersonal alude al sistema del núcleo modal, es decir, la representación del intercambio de bienes y servicios o información mediante el tipo de cláusula, la modalidad y la polaridad; y la metafunción textual apunta a la cláusula como mensaje estructurado en términos de progresión temática y de mecanismos de cohesión.

\subsection{La concepción de la gramática desde una perspectiva sistémico funcional}

La gramática se ha conceptualizado como un saber hacer con la lengua y un saber teórico sobre el funcionamiento de la lengua. La primera acepción apunta al desarrollo de la competencia comunicativa. La segunda, a un quehacer sistemático orientado a una descripción sobre las regularidades y principios que posibilitan la formación de estructuras bien formadas, para lo cual se requiere la distinción de las unidades significativas y sus posibilidades de combinación (Eguren \& Fernández Soriano, 2004; Di Tullio, 2007).

Desde la perspectiva formal, la gramática está centrada en la propiedad de los sistemas de reglas que posibilitan las combinaciones sintagmáticas de las unidades en los niveles morfológico y sintáctico. En esta línea, se postulan descripciones estructurales que explicitan tanto las unidades como las relaciones establecidas entre ellas, dándole un rol central a la sintaxis. Toda descripción estructural supone relaciones jerárquicas entre los constituyentes que determinan el comportamiento sintáctico entre elementos asimétricos que se dan en un tipo de sintagma (Chomsky, 1999; Eguren \& Fernández Soriano, 2004).

Desde una perspectiva funcional, se conciben las cadenas lingüísticas como interrelaciones entre el plano del contenido, el plano de la expresión y la función. En el plano de contenido, se da una interacción natural entre el nivel semántico y el 
léxico-gramatical, cuya organización se establece en términos sistémicos, es decir, como conjuntos de alternativas paradigmáticas. Esto supone que la gramática corresponde a una interface entre el mundo de la experiencia y la realización del significado. En esta línea, una secuencia sintagmática manifiesta las elecciones efectuadas para darle forma a los eventos y a las relaciones con otros. En el plano de la expresión, el contenido organizado léxico-gramaticalmente se expresa fonética y fonológicamente. Congruentemente, un elemento lingüístico es funcional en la medida que desempeña un rol central o un rol periférico para expandir el elemento central de una configuración sintagmática (Halliday \& Mathiessen, 2004).

En la lingüística sistémico-funcional, gramática y léxicos son partes de un continuo orientado a la producción de significado; sus relaciones son de complementariedad o, más concretamente, dos modos de aproximación a un mismo fenómeno. De acuerdo con Halliday y Matthiessen (1999), una gramática concebida como la descripción de patrones sistémicos es una teoría de la experiencia humana, una serie de recursos para reflexionar y actuar en el mundo.

Una gramática es sistémica cuando se la representa como un conjunto de redes en el orden paradigmático, es decir, como conjunto de alternativas para la construcción de significado con las condiciones que las caracterizan y viable de ser descrita en términos estructurales como sintagmas cuyas partes poseen funciones distintivas. Así, la gramática se enfoca desde el nivel semántico y sus componentes se describen en términos de sus interrelaciones funcionales, explicando el rol que desempeña cada elemento en la configuración orgánica del todo (Halliday \& Mathiessen, 2004).

\subsection{La cláusula como representación de la experiencia}

La cláusula es una configuración de elementos articulados para producir significados y que, prototípicamente, gira en torno a un centro nuclear realizado por un verbo o grupo verbal. Según Thompson (2014), la cláusula corresponde a cualquier segmento lingüístico cuyo núcleo es un grupo verbal finito (conjugación que le confiere finitud temporal y modal) o no-finito (forma nominal) y que posee una serie de posiciones funcionales, las que pueden ser llenadas por grupos de palabras con cierto tipo de cualidades estructurales. Como unidad mayor del estrato léxicogramatical, la cláusula está constituida por palabras o grupos de palabras que cumplen diversas funciones en los sistemas transitivo, interpersonal y textual (Martin, Matthiessen \& Painter, 1997; Halliday \& Mathiessen, 2004). Esto quiere decir que toda cláusula es una estructura multidimensional, pues en ella se realizan significados relacionados con la representación de la experiencia, las relaciones lógicas entre eventos, el intercambio con los otros y la organización del mensaje.

A nivel léxico-gramatical, se considera la cláusula como la unidad de procesamiento central, pues en ella se concretan las elecciones del hablante en diversas 
redes sistémicas y se las estructura sintagmáticamente. Cada uno de sus constituyentes realiza una función dentro del todo que se opone distintivamente con la de los otros con los cuales se articula. Por ejemplo, una cláusula como 'mi papá escondió los regalos en la casa de mi tía', desde el sistema de la transitividad pone en relación cuatro grupos de palabras: el verbo que representa el proceso representado y dos participantes inherentes que constituyen, junto con el proceso, el centro experiencial de la cláusula: 'mi papá' como el actor del proceso y 'los regalos' que constituye la meta, el objeto afectado por el proceso; dicho centro experiencial se localiza circunstancialmente 'en la casa de mi tía'. Interpersonalmente, 'mi papá' y el morfema gramatical ligado del verbo '-ió' constituyen el núcleo modal de la cláusula, en el cual el primero realiza la función de sujeto y el segundo la del finito que sitúa la cláusula en términos de tiempo (pasado) y modalización (alto grado de certeza); el resto constituye el residuo conformado por el predicador (la raíz verbal 'escond-), el objeto directo y un adjunto circunstancial. Textualmente, el grupo nominal 'mi papá' realiza la función de tema tópico y 'escondió los regalos en la casa de mi tía' la función de rema.

Desde el punto de vista de la representación de la experiencia, cada quantum de cambio se modela como una figura consistente en un proceso (verbo o grupo verbal) que se despliega temporalmente y con el cual se relacionan los participantes (realizado por grupos nominales) directamente involucrados como actor y meta de dicho proceso. Este centro experiencial (proceso + participantes) se puede expandir o proyectar por un atributo circunstancial. De este modo, la experiencia se configura como un conjunto manejable de procesos (Halliday \& Matthiessen, 1999; Halliday \& Matthiessen, 2004; Gutiérrez, 2015).

Una primera distinción de los tipos de proceso se relaciona con lo que se experimenta como algo externo (p. materiales), lo que se vivencia en el mundo de la conciencia (p. mentales) y las relaciones de un fragmento de la experiencia con otro en términos de clasificación e identificación (p. relacionales). Para explicar los componentes de cada proceso, se utilizará la traducción de los términos realizados por Chamorro, Barletta y Mizuno (2013).

En las cláusulas materiales (hacer y suceder en el mundo externo), un 'actor' tiene la fuerza para iniciar el proceso que recae sobre una 'meta' (el objeto afectado). En el ejemplo: 'mi papá escondió el regalo en la casa de mi tía', el participante 1 'mi papá' es el productor del proceso 'esconder' que recae sobre 'el regalo' que corresponde al participante 2 sobre el que recae la acción.

En las cláusulas mentales, 'el sensor' denota un ser consciente que realiza el proceso (sentir, pensar, querer, percibir) en relación con un contenido denominado 'el fenómeno'. El perceptor puede representar a una persona, a un colectivo humano o un producto de la consciencia humana (por ejemplo, 'el cuento imagina un mundo peligroso'). En el ejemplo: 'mi papá pensó esconder el regalo en la casa de mi tía', el 
participante 1 'mi papá' es quien experimenta el proceso de 'pensar', cuyo contenido es esconder el regalo.

En las cláusulas relacionales, se vinculan dos entidades por relaciones de identidad o clase-miembro. En concreto, el participante 1 ('el portador') posee (tener) o es igual (ser) a un participante 2 'el atributo'). Si el participante 1 se adscribe a una clase constituida por el participante 2 , se dice que es una cláusula relacional atributiva; en cambio, si el participante 1 es igual al participante 2 de tal modo que la relación entre ambos es reversible, se dice que es una cláusula relacional identificante. En el ejemplo, 'mi papá es el que escondió el regalo en la casa de mi tía' el participante 1 es el mismo que el participante 2, realizado por un grupo nominal en el que se incrusta una cláusula material.

Aparte de estos tres procesos principales, Halliday y Mathiessen (2004) identifican tres procesos subsidiarios: los verbales localizados en los límites entre lo relacional y lo mental; los conductuales en el límite entre lo mental y lo material; y los existenciales en el límite entre lo material y lo relacional.

En las cláusulas verbales (procesos del decir), el participante 1 corresponde a 'un hablante', que denota un ser consciente o una fuente simbólica (una investigación, una señal de tránsito, etc.), y el participante 2 'la verbalización' proferida, que puede realizarse como una cita textual o un reporte indirecto de lo dicho. En el ejemplo: 'mi papá reconoció que él había escondido el regalo', el participante 1 manifiesta un proceso del decir una verbalización proyectándola como un reporte realizado en una cláusula subordinada.

En las cláusulas conductuales, ligadas a comportamientos fisiológico y psicológico (respirar, toser, reír, soñar, descansar, escuchar) que son parcialmente mentales y parcialmente materiales o que representan un acto mental como un comportamiento (estar pensando), un 'ser consciente' manifiesta el proceso sin tener necesariamente la intención de hacerlo, como una reacción a un estímulo. En el ejemplo: 'mi papá se ríe después de esconder el regalo', el participante 1 'mi papá manifiesta el comportamiento de reír como efecto de la acción realizada en la cláusula no-finita.

En las cláusulas existenciales, se indica el rasgo de 'existente' de un evento o entidad, lo cual desempeña un importante rol para introducir un participante en un texto. Por ejemplo, 'mi papá estaba en casa de mi tía' (participante + ser/estar + circunstancia), 'había un regalo en casa de mi tía' (haber impersonal + participante).

\section{Metodología}

La investigación se enmarca en un enfoque cuantitativo de nivel descriptivo (Hernández Sampieri, Fernández Collado \& Baptista Lucio, 2010). Para tal efecto, se trabajó con una muestra de estudiantes de dos escuelas con niveles socioeconómicos 
diferentes según la clasificación del Ministerio de Educación (Mineduc). La primera es una escuela particular subvencionada de la comuna de Santiago (NSE medio alto) y la segunda una municipal de la comuna de Estación Central (NSE medio bajo). Ambas escuelas cuentan con resultados sobre el promedio regional en las pruebas aplicadas por el Sistema de Medición de la Calidad Educativa (Simce) y el propósito era explorar la existencia de posibles diferencias en el uso de recursos lingüísticos entre los estudiantes de estos dos tipos de establecimientos. Los estudiantes cursaban el $3^{\circ}, 5^{\circ}$ y $7^{\circ}$ años. Se partió con el $3^{\circ}$ básico, puesto que se espera que en dicho nivel los estudiantes hayan adquirido el código escrito. De allí, se estableció una diferencia de dos años, con el objeto de apreciar con mayor claridad los posibles cambios a medida que se avanza en la escolaridad.

Se desarrolló una tarea de escritura consistente en una narración espontánea sobre una experiencia personal libremente elegida, según un guion validado por un criterio de jueces (Rubio, 2016). Dicha tarea partió por conversar en cada curso sobre la labor que desarrollan los escritores $y$, luego, generar las condiciones para que individualmente recordaran una experiencia vivida que quisieran contar a otros. Estando los niños con los ojos cerrados, se les orientó para que visualizaran detalles sobre lo ocurrido, las acciones efectuadas, las personas involucradas y las emociones experimentadas. Finalmente, se les solicitó que escribieran la experiencia recordada con el fin de que dicho relato fuera leído por otros.

Esta tarea fue aplicada por los profesores de lenguaje de cada establecimiento como una actividad propia de la asignatura y con la presencia del investigador. El plazo máximo para la realización de la tarea fue de 80 minutos. En total, se recogieron 133 textos en el establecimiento particular subvencionado y 142 textos en el municipal. De estos, se eligió una muestra al azar de 90 textos por establecimientos, con una cuota igual de 30 por cada curso. En total, se analizaron los textos escritos por 180 escolares.

Se efectuó una segmentación clausular de cada texto para identificar los tipos de procesos representados. En primer lugar se segmentó el propio texto en complejos clausulares y cláusulas simples siguiendo la convención indicada por Halliday y Mathiessen (2004). 
A medida que se avanza en la escolaridad, los estudiantes escriben textos con un mayor número de cláusulas (16,48; 21,35 y 35.77 promedio de cláusulas por textos), aunque también la variabilidad al interior de los cursos es mayor (desviación estándar 8,97; 10,98 y 20,9 respectivamente). Entre los dos tipos de establecimientos, la frecuencia de cláusulas por textos es relativamente semejante $(25,65$ promedio en la escuela municipal y 23,42 en la particular subvencionada), aunque la variabilidad es mayor en la municipal (DS 19,36) que en la particular subvencionado (DS 13,46).

El primer análisis se orientó a la identificación de procesos representados en las cláusulas de cada texto con el fin de establecer cuáles eran los predominantes. Posteriormente, se hizo un análisis más detallado de los materiales, los relacionales y los mentales que fueron los que obtuvieron mayor frecuencia. Los porcentajes promedio de los procesos representados en los textos de los estudiantes se presentan en el Gráfico 1.

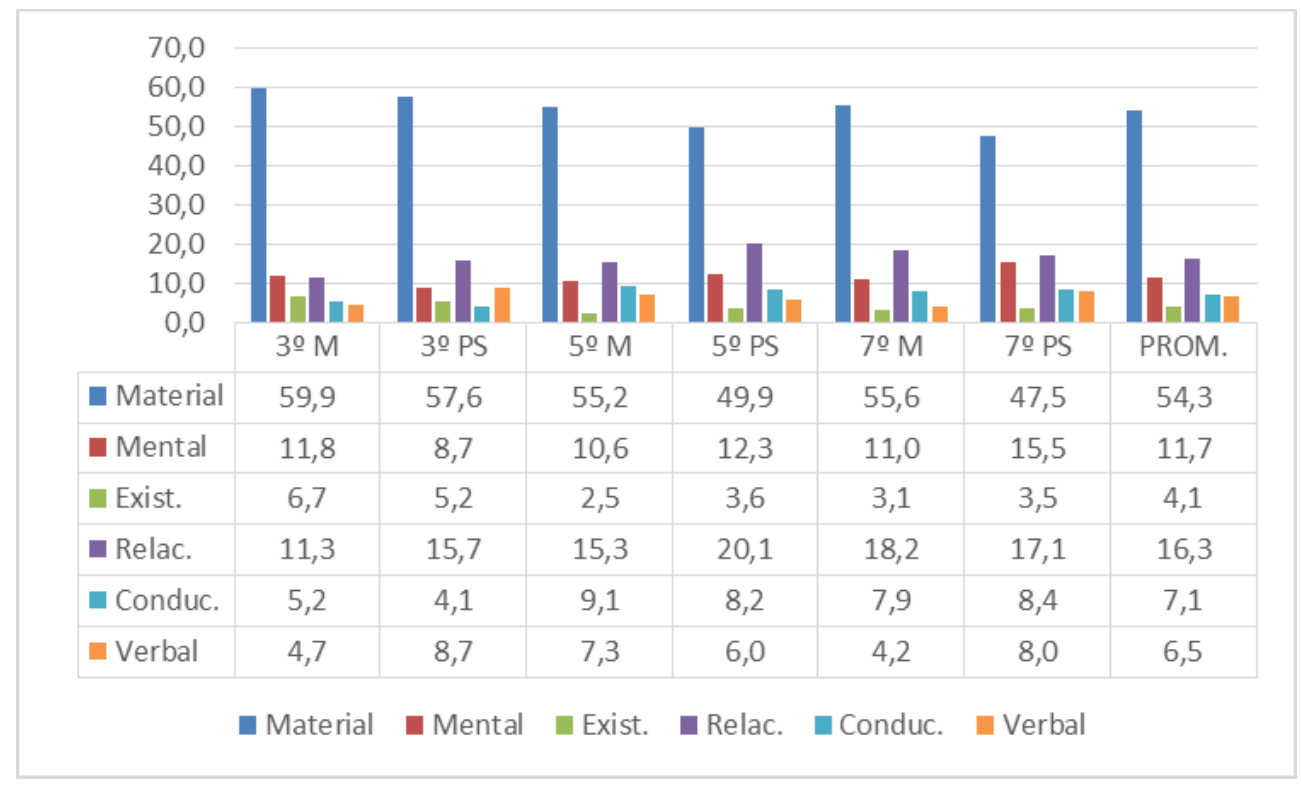

Gráfico 1. Porcentajes de procesos representados en las cláusulas que componen los textos de los estudiantes, distribuidos por nivel y tipo de establecimiento.

Al narrar una experiencia personal, los escolares tienden a escribir textos en los que predominan sustantivamente cláusulas que expresan procesos materiales. Le siguen con una frecuencia mucho menor las cláusulas relacionales y las mentales. La menor frecuencia está dada por las cláusulas con procesos conductuales, verbales y existenciales. En otras palabras, los escolares tienden a localizar la experiencia como un conjunto de acciones y sucesos que se experimentan en el mundo externo. Además, se enfatiza la descripción de las relaciones entre entidades observadas en términos de pertenencia a una clase o identificación. 
Dado que los procesos materiales tienen una frecuencia sustantivamente mayor en los textos escritos por los estudiantes, partiremos por un análisis más detallado de estas. Posteriormente, continuaremos con una visión más general de las cláusulas que expresan procesos relacionales y mentales.

Es importante destacar que, al comparar la ocurrencia de procesos materiales en los escritos de los estudiantes, prácticamente no hay diferencias significativas según la modalidad del establecimiento (p: $0.061>0.05$ ) ni según el nivel cursado (p: $0.079>$ 0.05).

En general, los estudiantes de los tres niveles de ambos establecimientos escriben cláusulas materiales operativas $(99,7 \%)$, las que reflejan situaciones concretas $(99,6 \%)$ y sobre eventos que ocurren en la realidad $(96,1 \%)$, es decir, que no implica un quehacer creativo impulsado por ellos para transformar un objeto. En otras palabras, el actor, entendido como una entidad concreta, realiza un evento vinculado con el desplazamiento (ir, entrar, avanzar, volver, subir, llegar, bajar, escalar, andar en bicicleta), el despliegue de una acción (jugar, comprar, regalar, esconderse, comer, vivir, encontrar, pescar, bailar) o sufrir un inconveniente (caer, resbalar, golpear, operarse). La mayor cantidad de las cláusulas materiales son intransitivas $(71,9 \%)$, es decir, el proceso no recae sobre un objeto u actividad concreta. Respecto de este tema, en la escuela municipal no hay un patrón claro (el $75 \%$, el $64,4 \%$ y el $76 \%$ en $3^{\circ}, 5^{\circ}$ y $7^{\circ}$ básicos respectivamente). Por el contrario, en la escuela municipal existe una diferencia sustantiva en la frecuencia de cláusulas intransitivas entre $3^{\circ}$ y $5^{\circ}$ básicos ( $81,1 \%$ y $67,3 \%$ respectivamente), pero no hay cambios entre el $5^{\circ}$ y el $7^{\circ}$ básico $(67,4 \%)$.

Si consideramos el porcentaje de cláusulas materiales por textos, el texto del estudiante que contiene menos cláusulas de este tipo es de 12,5\%. Por el contrario, el texto que contiene mayor porcentaje de este tipo alcanza el 100\%. Al construir un índice con esta información con el propósito de apreciar el grado de orientación del relato hacia los eventos externos, se generaron cinco categorías (ver Tabla 2), cuyo rango se estableció tomando como parámetro la desviación estándar del total de la muestra (17,5 puntos porcentuales de variación). 
Tabla 2. Porcentaje de sujetos distribuidos según la orientación de su relato hacia la representación de procesos materiales (externalización de la experiencia personal).

\begin{tabular}{|l|c|c|c|c|c|c|}
\hline \multirow{2}{*}{ Orientación del relato } & \multicolumn{3}{|c|}{ Escuela municipal } & \multicolumn{3}{c|}{ Escuela particular subvencionada } \\
\cline { 2 - 7 } & $\mathbf{3}^{\mathbf{0}}$ & $\mathbf{5}^{\mathbf{0}}$ & $\mathbf{7}^{\mathbf{0}}$ & \multicolumn{2}{|c|}{$\mathbf{3}^{\mathbf{0}}$} & \multicolumn{2}{|c|}{$\mathbf{5}^{\mathbf{0}}$} & $\mathbf{7}^{\mathbf{0}}$ \\
\hline $\begin{array}{l}\text { Interna } \\
(12.5 \%-30.0) \%\end{array}$ & 6.66 & 6.66 & 6.66 & 10.0 & 16.66 & 10.0 \\
\hline $\begin{array}{l}\text { Relativamente interna } \\
(30.1 \%-47.5 \%)\end{array}$ & 26.66 & 20.0 & 30.0 & 20.0 & 26.66 & 36.66 \\
\hline $\begin{array}{l}\text { Neutra } \\
47.6-65.1\end{array}$ & 30.0 & 43.33 & 30.0 & 20.0 & 30.0 & 40.0 \\
\hline $\begin{array}{l}\text { Relativamente externa } \\
(65,2 \%-82,7 \%)\end{array}$ & 20.0 & 30.0 & 26.66 & 46.66 & 26.66 & 6.66 \\
\hline $\begin{array}{l}\text { Externa } \\
(82,2 \%-100 \%)\end{array}$ & 16.66 & 0.0 & 6.66 & 3.33 & 0.0 & 6.66 \\
\hline Total & 100 & 100 & 100 & 100 & 100 & 100 \\
\hline
\end{tabular}

En la escuela municipal, es posible observar que existe la tendencia a la disminución progresiva de la orientación de externalización de la experiencia narrada a medida que se avanza en la escolaridad. Dicha tendencia aparece más claramente definida en la escuela particular subvencionada.

En relación con los participantes, los textos de los estudiantes, de ambos establecimientos y de los tres niveles, tienden a construir cláusulas materiales con un actor explícito o fácilmente recuperable del cotexto (97,3\%). El 26,4\% de las cláusulas expresa la meta del proceso y el $8 \%$ su destinatario. En general, no se aprecia un patrón de variación sustantiva entre ambos establecimientos y entre los diferentes niveles.

Cabe indicar que el $60,1 \%$ de las cláusulas materiales presentan una o más elementos circunstanciales. En términos de los sujetos, el 98,33\% explicita, a lo menos, un elemento circunstancial en que tal proceso ocurre. Al considerar los textos según tipo de establecimiento, se constata que los estudiantes de la escuela municipal tienden a ser significativamente más descriptivo respecto del escenario en que ocurren los procesos materiales narrados (p: $0.0085<0.05$ ). En términos de los cursos, no hay diferencias significativas en la medida que se avanza en la escolaridad (p: $1.0387>$ 0.05). En la escuela municipal, el porcentaje de cláusulas materiales con elementos circunstanciales es de $67,4 \%$ en $3^{\circ} ; 60,6 \%$ en $5^{\circ}$ y $64 \%$ en $7^{\circ}$; en la escuela particular subvencionada, los porcentajes son bastante estables $(57,1 \%, 56 \%$ y 55,6\%, respectivamente).

A continuación, se presentan el tipo de elementos circunstanciales con mayor prevalencia en los textos escritos por los escolares (ver Gáfico 2). 


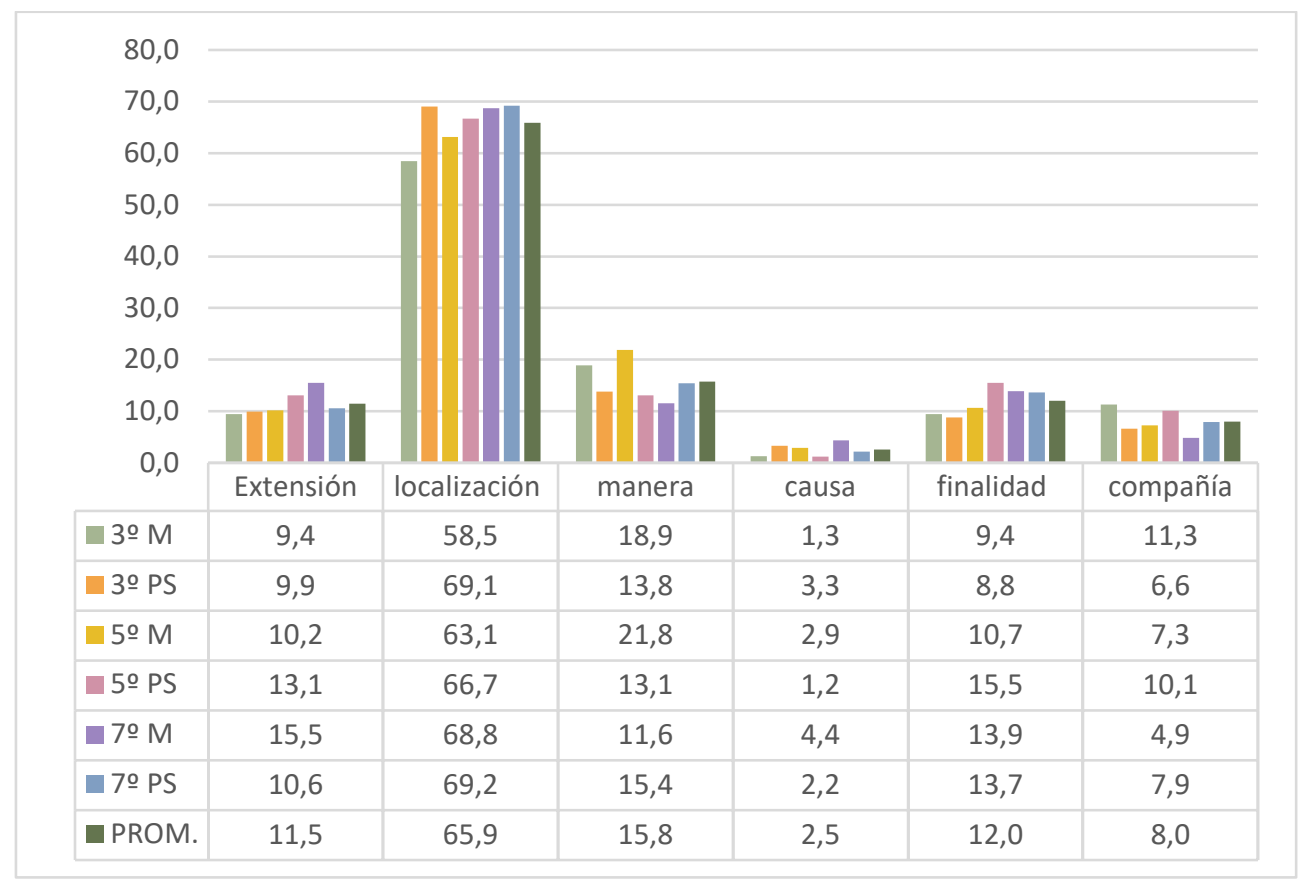

Gráfico 2. Tipos de elementos circunstanciales en las cláusulas de procesos materiales, indicada en porcentaje.

Los estudiantes de los tres niveles, en ambos establecimientos, privilegian la descripción de circunstancias relativas a la ubicación temporo-espacial de los eventos materiales representados. En la escuela municipal, existe una clara tendencia a un incremento sostenido de la identificación del lugar y el tiempo en que ocurre el proceso; mientras que en la escuela particular subvencionada es posible apreciar una relativa estabilidad de la descripción de estos aspectos circunstanciales. De todos modos, entre los dos tipos de establecimientos no hay diferencias significativas respecto de este tipo de elemento circunstancial (p: $0.664>0.05$ ). Al considerar la variable curso, tampoco se observan diferencias significativas (p: $2.01>0.05)$.

Fuera de la localización, la mayor frecuencia de circunstancias con que se describen los eventos son las de manera, finalidad y extensión. Son los estudiantes de la escuela municipal los que más destacan el modo en que se desarrolla el proceso, especialmente en $3^{\circ}$ y $5^{\circ}$ básicos. Por su parte, son los estudiantes de niveles superiores los que tienden a ser más explícitos en las finalidades de los procesos representados. En relación a las relaciones de causalidad, si bien la expresión de estas circunstancias es bastante baja, se aprecia en los estudiantes de la escuela municipal la tendencia creciente a explicitarlas.

Respecto de las cláusulas relacionales, se puede sostener que la tendencia es que en los escritos de los estudiantes haya más cláusulas relacionales de identificación $(58,5 \%)$ 
que de atributo $(41,5 \%)$. Esto implica que los estudiantes se concentrarían más en describir la pertenencia de un objeto a una clase que a la explicitación de sus características. No obstante, a medida que los estudiantes avanzan en la escolaridad (en ambos establecimientos) se tiende a describir más las características de los objetos representados y a darle menos importancia a su clasificación (ver Gráfico 3). En este sentido la adjetivación calificativa sería un aspecto central.

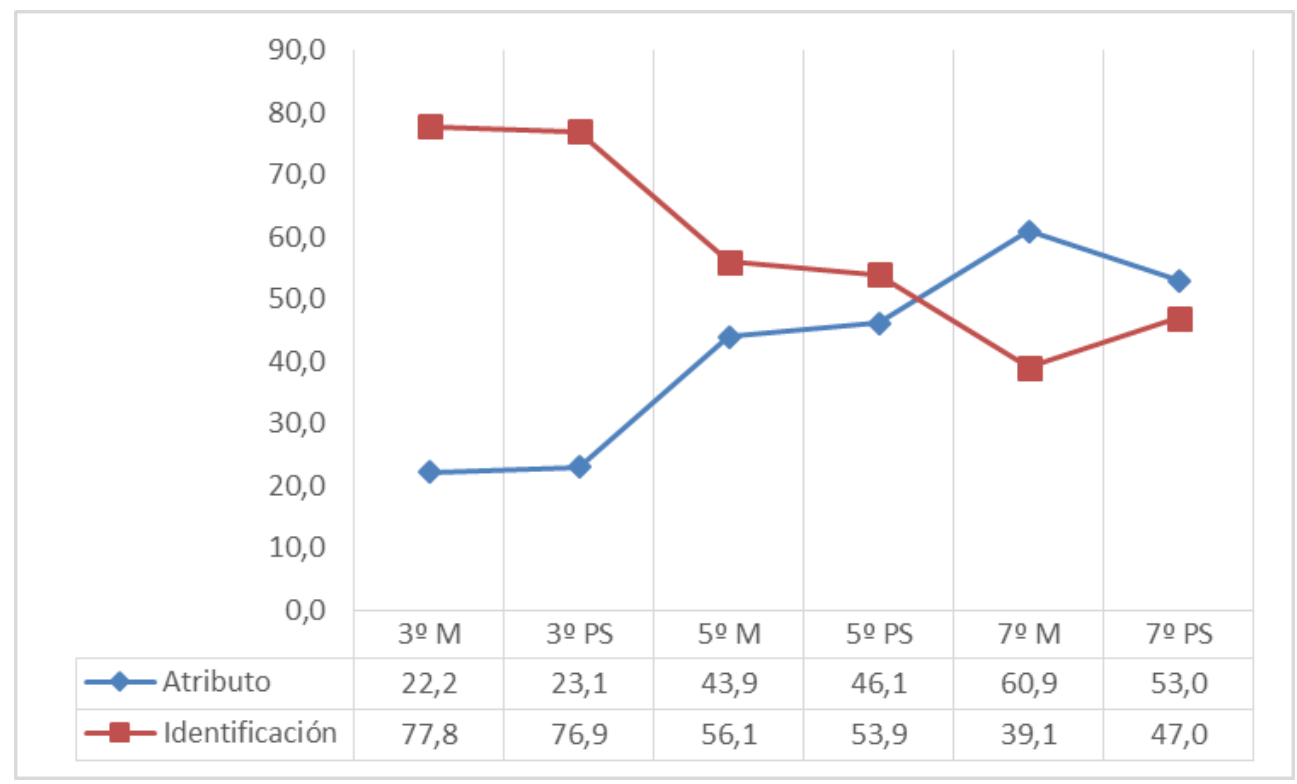

Gráfico 3. Porcentaje de tipo de cláusulas relacionales en los textos de estudiantes por curso y modalidad del establecimiento.

En relación con las cláusulas mentales, la mayoría de los sujetos de todos los niveles expresa procesos relacionados con la conciencia y esta tendencia tiende a aumentar a medida que se avanza en la escolaridad. Aunque, como se observa en el Gráfico 4, en la escuela particular subvencionada existe un patrón de incremento sostenido; en cambio, en la municipal, se observa un incremento relevante al comparar el porcentaje de sujetos de $3^{\circ}$ básico con los de $5^{\circ}$, no así entre el $5^{\circ}$ y el $7^{\circ}$ básico. 


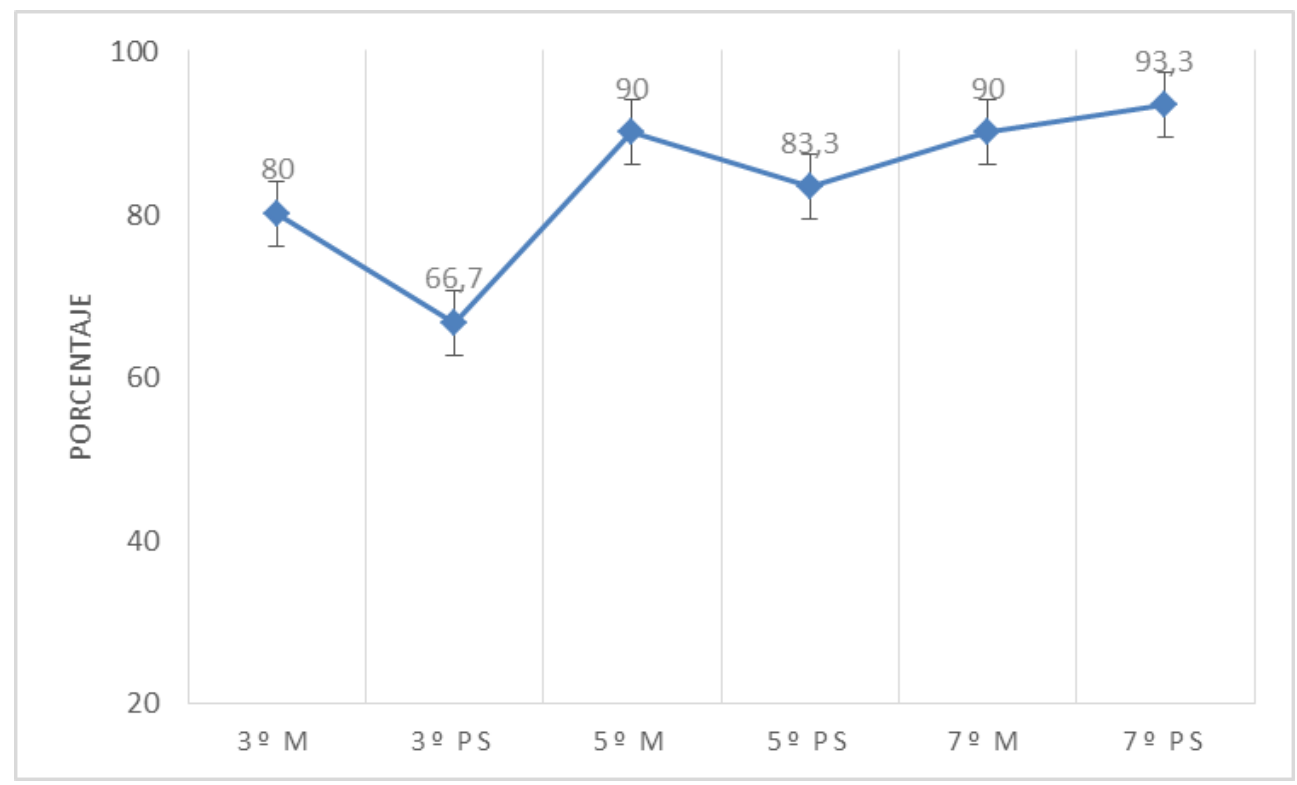

Gráfico 4. Porcentaje de sujetos cuyos textos presentan cláusulas mentales, según nivel y modalidad del establecimiento.

Al precisar el tipo de proceso mental, se constata que los estudiantes de todos los niveles, de ambos establecimientos, tienden a representar procesos de orden afectivo $(45,5 \%)$ cuando sitúan un evento a nivel de la conciencia. Con una frecuencia sustantivamente menor, le siguen los procesos mentales de cognición (28\%) y los de percepción (26,5\%). A medida que se avanza en la escolaridad, tiende a descender el porcentaje de procesos afectivos, tendencia que es mucho más clara al comparar los $3^{\circ}$ con los $5^{\circ}$ básicos. Paralelamente, tienden a tener un incremento gradual los relativos a la cognición (ver Gráfico 5). 


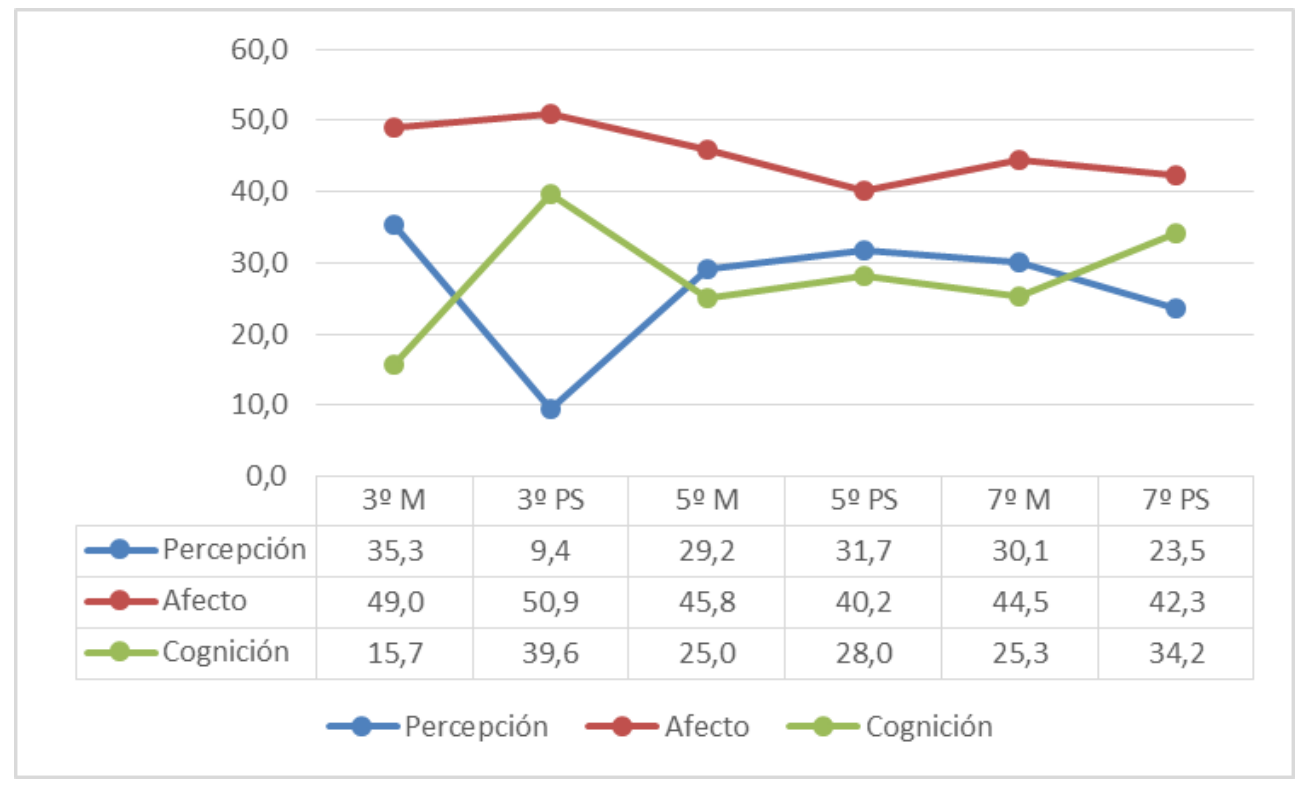

Gráfico 5. Porcentaje de tipos de cláusulas mentales presentes en los textos escritos por los estudiantes, según nivel y modalidad del establecimiento.

Cabe destacar que los estudiantes de la escuela municipal tienden a escribir un mayor número de cláusulas con procesos mentales de percepción, especialmente ligadas a la vista. Por su parte, los de la escuela particular subvencionadas tienden a privilegiar más las relativas a la cognición, es decir, con el saber, imaginar, aprender, enseñar, investigar.

En relación con los procesos verbales, un 52,8\% de los escolares explicita en sus textos al menos una cláusula con este tipo de proceso. Al cruzar este dato, con la modalidad del establecimiento es posible constatar que el 73\% de los escolares del establecimiento particular subvencionado incluye algunas cláusulas con procesos verbales, mientras que en el establecimiento municipal solo lo hace el 33,3\% de los sujetos. Al respecto, la tendencia en la escuela particular subvencionada es a un incremento significativo desde el $5^{\circ}$ al $7^{\circ}$ año; en cambio, el patrón en la escuela municipal es menos claro (ver Gráfico 6). 


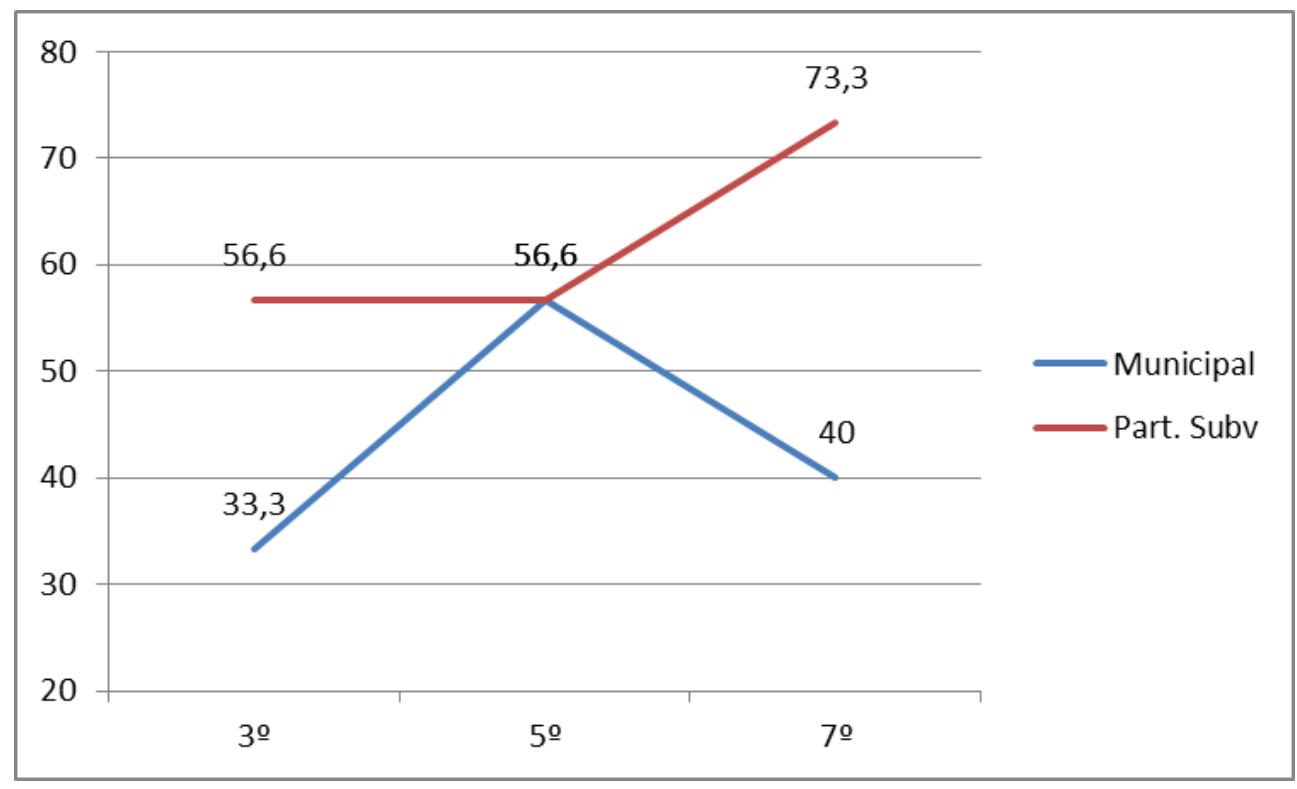

Gráfico 6. Sujetos cuyos textos presentan cláusulas verbales, expresada en porcentaje según tipo de establecimiento y nivel de escolaridad.

Los resultados obtenidos implican que, a medida que se avanza en la escolaridad, los textos de los estudiantes se complejizan en cuanto a su extensión, el despliegue de recursos para la localización y descripción de los eventos y el tránsito hacia una orientación progresivamente más interna de la representación de la experiencia. Dicho hallazgo es coherente con estudios que evidencian que la escolaridad es un factor relevante en el dominio creciente de recursos gramaticales. Esto estaría en relación con el impacto que la edad y la escolarización tiene sobre los modos de procesamiento de la experiencia y, congruentemente, con las formas de organizarla lingüísticamente (Benítez, 2009; Aravena, 2011; Aravena \& Hugo, 2016; Iparraguirre et al., 2016). En otras palabras, un cambio fundamental, concordante con los datos expuestos, es que la escolaridad permite avanzar desde un enfoque centrado en la estructura de los eventos hacia la interpretación de los mismos (Aravena, 2011). Finalmente, los resultados obtenidos son consistentes con estudios que muestran que, el dominio progresivo de recursos discursivos en la escolaridad, favorece la capacidad de localizar contextualmente las acciones narradas (Iparraguirre et al., 2016).

\section{CONCLUSIONES}

Al describir los procesos presentes en las narraciones ligada a la experiencia personal escritas por escolares básicos, es posible identificar un predominio sustantivo de las cláusulas finitas que representan procesos materiales, es decir, representan a actores realizando eventos observables en un marco temporo-espacial claramente especificado. Dichos procesos fundamentalmente se relacionan con el desplazamiento, 
la concreción de una acción o situaciones accidentales. En otras palabras, los textos escritos por los escolares se caracterizan por la tendencia a describir eventos concretos claramente perceptibles desde una perspectiva externa con un actor claramente identificable. A pesar de que no se evidencian diferencias significativas, considerando las variables curso y tipo de establecimiento, en el predominio de dichas cláusulas en los textos de los escolares, sí es posible establecer que hay diferencias al analizar los escritos de cada sujeto. En esta línea, el avance en la escolaridad haría que cada estudiante fuera incorporado una mayor frecuencia de cláusulas mentales -cuyo incremento sería más notable entre el $3^{\circ}$ y el $5^{\circ}$ básico- y cláusulas relacionales atributivas explicitando así las características de los sujetos y eventos representados.

En relación con las cláusulas mentales, tienden a predominar aquellas que expresan afecto, aunque se verifica una tendencia a una progresiva disminución de estas a medida que se avanza en edad. En este sentido, con la escolaridad se irían incrementando las cláusulas mentales de percepción y cognición, cambio que sería mucho más claro al comparar el $3^{\circ}$ con el $5^{\circ}$ básico.

En concordancia con el marco teórico, las narraciones sobre la experiencia personal de los escolares se ciñen a los tres procesos considerados como principales: materiales, relacionales y mentales, aunque predomina claramente una visión externalizadora de los procesos experimentados. $\mathrm{Al}$ respecto, es posible hipotetizar que el predominio de la representación de los eventos como algo externo se deba, por un lado, al efecto de la modalidad discursiva elegida en la tarea y, por otro, a las prácticas de lectura de narraciones en la escuela básica que ponen el acento en la acción más que en la reflexión y calificación de lo vivido. De todos modos, es importante destacar que la escolaridad es un factor que incidiría en el despliegue de procesos mentales cognitivos y recursos para la asignación de atributos a la experiencia vivida.

De acuerdo con los datos analizados, también es posible esperar que con el avance en la escolaridad los estudiantes vayan incorporando en su relato cláusulas que representen procesos verbales. En este sentido, llama la atención la existencia de un gran porcentaje de estudiantes en la escuela municipal que representan sujetos que no se expresan verbalmente. Pareciera ser que a las personas le ocurren cosas y reaccionan frente a ellas en silencio.

En relación a la exploración de la incidencia del factor tipo de establecimiento, se constata que las diferencias se dan en: la variabilidad existente en la extensión de los textos al interior del establecimiento municipal, la permanencia del porcentaje de sujetos que escriben textos con una orientación externa en este tipo de establecimiento frente a la disminución sostenida de dicha orientación en el particular subvencionado y al poco incremento de estudiantes del establecimiento municipal que incorporan en sus relatos cláusulas verbales. No obstante, los estudiantes de 
establecimientos municipales tenderían a una mayor caracterización de los eventos narrados.

En síntesis, en educación básica es posible esperar la producción de narraciones escritas con un predominio de procesos materiales, aunque se puede establecer como un desarrollo potencial para los estudiantes, a medida que avanzan en la escolaridad, la configuración de narraciones en que se vaya incrementando gradualmente la reflexión y valoración de los eventos representados, proporcionando recursos lingüísticos a los estudiantes para introducir procesos mentales vinculados con la cognición, procesos relacionales atributivos y procesos verbales que expresen mediante citas o reporte indirecto la interacción de los actores.

Para profundizar la descripción presentada, habría que explorar el tipo de procesos representados por los escolares básicos cuando escriben otros tipos de textos y analizar los cambios que se registran con la escolaridad de otros tipos de recursos lingüísticos. Asimismo, sería relevante estudiar con mayor profundidad la incidencia del factor socioeconómico en el uso de los recursos lingüísticos, pues así se dispondrían de insumos para el diseño de intervenciones didácticas en el ámbito de la producción escrita.

\section{REFERENCIAS BIBLIOGRÁFICAS}

Alegría Ugarte, M. \& Díaz Agüero, C. (2017). ¿Se escribe junto o separado?: Evolución en el uso de los espacios en blanco en textos de niños que inician su alfabetización. Revista Signos. Estudios de Lingüística, 50(93), 3-25.

Aravena, S. (2011). El desarrollo narrativo a través de la adolescencia: Estructura global de contenido y referencia personal. Revista Signos. Estudios de Lingüistica, $44(77), 215-232$.

Aravena, S. \& Hugo, E. (2016). Desarrollo de la complejidad sintáctica en textos narrativos y explicativos escritos por estudiantes secundarios. Lenguas Modernas, 47, 9-40.

Aravena, S., Figueroa, J., Quiroga, R. \& Hugo, E. (2016). Organización discursiva de dos géneros en estudiantes de tres niveles de escolaridad y diferentes grupos sociales. Revista Signos. Estudios de Lingüistica, 49(91), 168-191.

Benítez, R. (2009). Análisis descriptivo de narraciones escritas por niños y niñas de tercer año básico. Literatura y Lingüística, 20, 103-123.

Benítez, R. \& Sotelo, E. (2013). Calidad de la producción escrita en dos secuencias textuales según tipo de establecimiento educacional. Logos, 23(2), 127-150. 
Chamorro, D., Barletta, N. \& Mizuno, J. (2013). El lenguaje para enseñar y aprender las Ciencias Naturales: Un caso de oportunidades perdidas para la formación ciudadana. Revista Signos. Estudios de Lingüística, 46(81), 3-28.

Christie, F. \& Derewianka, B. (2010). School discourse. Londres: Continuum.

Chomsky, N. (1999). Aspectos de la teoría de la sintaxis. Barcelona: Gedisa.

Concha, S., Aravena, S., Coloma, C. J. \& Romero, V. (2010). Escritura expositiva en tres niveles de escolaridad: Coherencia y dominio de recursos lingüísticos. Literatura y Lingüística, 21, 75-92.

Crespo, N., Benítez, R. \& Pérez, L. (2010). Conciencia metapragmática y la habilidad para producir narraciones escritas. Revista Signos. Estudios de Lingüistica, 43(73), 179-209.

Di Tullio, A. (2007). Manual de gramática del español. Buenos Aires: La isla de la luna.

Eggins, S. (2003). Introducción a la lingüística sistémica. Logroño: Universidad de la Rioja.

Eguren, L. \& Fernández Soriano, O. (2004). Introducción a una sintaxis minimista. Madrid: Gredos.

Grabe, W. \& Kaplan, R. (2014). Theory and practice of writing. Nueva York: Routledge.

Gutiérrez, R. M. (2008). El género manual en las disciplinas académicas: Una caracterización desde el sistema de la obligación. Revista Signos. Estudios de Lingüística, 41(67), 177-202.

Gutiérrez, R. M. (2015). Transitividad en el español de Chile: Exploración desde la perspectiva sistémico-funcional. Revista Signos. Estudios de Lingüistica, 48(88), 223-249.

Halliday, M.A.K. (1998). El lenguaje como semiótica social. La interpretación social del lenguaje y del significado. México: FCE.

Halliday, M.A.K. (2003). On language and linguistics. Londres: Continuum.

Halliday, M.A.K. \& Matthiessen, C. (1999). Construing experience through meaning: A language-based approach to cognition. Londres: Cassell.

Halliday, M.A.K. \& Matthiessen, C. (2004). An introduction to functional gramar. Londres: Arnold.

Hayes, J. (1996). A new framework for understanding cognition and effect in writing. En C. M. Levy \& S. E. Ransdell (Eds.), The science of writing. Theory, methods, individual differences and applications (pp. 1-27). Mahwa, N. J.: Lawrence Erbaum. 
Hernández Sampieri, R., Fernández Collado, C. \& Baptista Lucio, P. (2010). Metodología de la investigación. México: McGraw Hill.

Iparraguirre, M. S., Baccala, N. \& Scheuer, N. (2016). Variaciones lingüísticodiscursivas en narrativas escritas por alumnos de nivel primario en Norpatagonia, Argentina. Revista Signos. Estudios de Lingüistica, 49(92), 304-328.

Lacon de Lucia, N. \& Ortega de Hocevar, S. (2008). Cognición, metacognición y escritura. Revista Signos. Estudios de Lingüistica, 41(67), 231-255.

Martin, J., Mathiessen, C. \& Painter, C. (1997). Working with functional grammar. Londres: Arnold.

Meneses, A., Ow, M. \& Benítez, R. (2012). Complejidad sintáctica: ¿Modalidad comunicativa o tipo textual? Estudio de casos de producciones textuales de estudiantes de $5^{\circ}$ básico. Onomáz̨ein, 25, 65-93.

Pinheiro, F. \& de Freitas Rossi, T. (2008). A consciência metalingüística pragmática e sua relação com a produção escrita. Revista de Lingüística Teórica y Aplicada, 46(2), 29-51.

Rose, D. \& Martin J. R. (2012). Learning to write, reading to learn. Genre, knowledge and pedagogy in the Sydney School. Sheffield: Equinox.

Rubio, M. (2016). Narraciones de escolares básicos: Dos tareas de escrituras. Literatura y Lingüistica, 34, 221-244.

Sotomayor, C., Molina, D., Bedwell, P. \& Hernández, C. (2013). Caracterización de problemas ortográficos recurrentes en alumnos de escuelas municipales chilenas de $3^{\circ}, 5^{\circ}$ y $7^{\circ}$ básico. Revista Signos. Estudios de Lingüistica, 46(81), 105 131.

Sotomayor, C., Lucchini, G., Bedwell, P., Biedma, M., Hernández, C. \& Molina, D. (2013). Producción escrita en la Educación Básica: Análisis de narraciones de alumnos de escuelas municipales de Chile. Onomázein, 27, 53-77.

Sotomayor, C., Gómez, G., Jéldrez, E., Bedwell, P., Dominguez, A. M. \& Ávila, N. (2016). Evaluación analítica de la escritura de estudiantes de $4^{\circ}$ año básico en Chile. Onomázein, 34, 381-425.

Thompson, G. (2014). Introducing functional grammar. Londres: Routledge. 


\section{ANEXO}

\section{Masculino}

5o básico

Particular Subvencionado

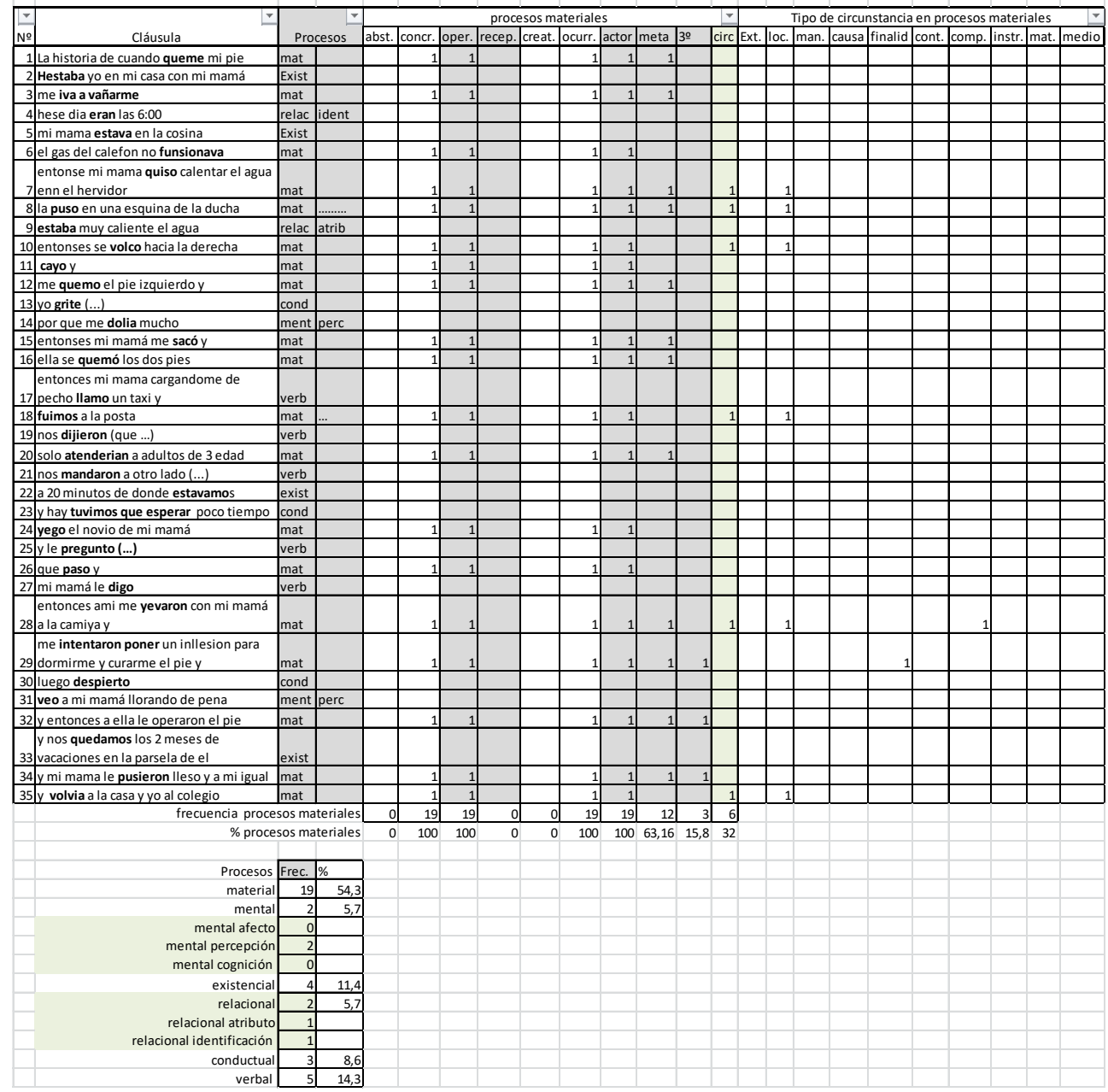

\section{NOTA}

1 Este trabajo forma parte del proyecto Fondecyt/iniciación 11130010: Desarrollo de la producción escrita en escolares básicos: un análisis desde la lingüística sistémico funcional. 